\title{
Dynamic Modeling of the Drying Process of Corn Grains using Neural Networks
}

\author{
Galih Kusuma Aji ${ }^{1 *}$, Wildan Fajar Bachtiar ${ }^{1}$, Henry Yuliando ${ }^{2}$, Endy Suwondo \\ ${ }^{1}$ Department of Bioresource Technology and Veterinary, Vocational College, Universitas Gadjah Mada, \\ Jl. Yacaranda, Sekip Unit II, Yogyakarta 55281, Indonesia \\ ${ }^{2}$ Department of Agro-industrial Technology, Faculty of Agricultural Technology, Universitas Gadjah Mada, \\ Jl. Flora No. 1 Bulaksumur, Yogyakarta 55281, Indonesia \\ *Email: ajikun@ugm.ac.id
}

Submission: March 25, 2019; Acceptance: July 11, 2019

\begin{abstract}
This study explored a dynamic modelling of corn drying process. The appropriate use of a dynamic model for the drying process of corn grains could lead to an effective method for optimizing the system. The optimal control strategy can be determined by predicting the future behaviors of the process using a dynamic model. In this work, the dynamic characteristic of the corn grains' water loss during a temperature dynamics treatment in the drying process was measured in a continuous manner using a precise load cell. The nonlinear autoregressive with external input (NARX) neural network was applied to identify and to develop a model of dynamic characteristics of the corn grains drying process. Then, for model training and validation, the dynamic responses of the corn grains' water loss rate to drying temperature were used. A three-layered NARX neural network model, which consists of the 1-10-1 neuron number of each layer with two times delay was successfully developed to identify and to make a model for such a complex system. The developed model showed the accuracy of the corn grains' water loss rate during the drying process with the mean square error $(M S E)$, and determination coefficient $\left(R^{2}\right)$ values of $1.89 \mathrm{x}$ $10-4$ and 0.89 consecutively.
\end{abstract}

Keywords: Drying temperature; neural network; drying process; dynamic model; water loss rate

\section{INTRODUCTION}

Drying has been widely known as a popular method to preserve agricultural grain product. In many cases, the environmental factors during the drying process of agricultural grains product are maintained constant. A constantly high temperature in the drying process is considered as a static system. Under this condition, however, it would be difficult to have an optimal process control. A controlled drain drying process is essential to prevent the grain loss, maintain the quality and enable to longer preservation of grain. Aini et al. (2017) reported that drying is an energy intensive and complex nonlinear process; therefore, it is difficult to control. In this study, we propose an alternative approach by identifying the drying process of grain as a dynamic system.
In order to apply optimal control to the drying process of grains, a dynamic model of the process is needed. The optimal control strategy can be specified by predicting the future behavior of the process using the dynamic model (Morimoto and Hashimoto, 2009). Model is also used for a better understanding of the process behavior and synthesis of the control system (Fasol and Jörgl, 1980). For realizing the dynamic model, measurement, and identification of the physiological responses of the grains are important. Through monitoring the weight of grains during the drying process, continuous and non-destructive measurement of the physiological response of the grains can be conducted.

However, it is hard to successfully construct the dynamic model physiological process of grains to 
environmental factor optimally based on conventional mathematical approaches characterized by deterministic parameters and mathematical equation. It is due to the physiological process that has real complex properties, such as strong nonlinearity, marked time-delay, and time-varying system parameters, and the response is characterized by complexity and uncertainty (Aghbashlo et al., 2015). The other method for constructing the dynamic model is system identification, which deals with the unknown process (Ljung, 2010). The identification methods have been widely used in food preservation (Baloch et al., 2006). Recently, artificial intelligent technique, such as neural networks, has been extensively developed in agricultural field (Morimoto et al., 2009) and postharvest grain process (Dai et al., 2017). This technique has proven to be powerful tools for dealing with complex problems to which conventional mathematical approaches cannot be applied. Neural networks are effective for dynamic model development of complex and fuzzy systems because they can well acquire the essential dynamics with its high learning capability (Rumelhart et al., 2013).

This paper proposes a model development technique based on neural networks on the drying process of corn grains system, with the control input variable is the drying temperature, and the output is physiological grains responses. The various study on the application of neural network for drying technology has been reported by (Aghbashlo et al., 2015). Thus this work also will add a new perspective in the approach of modeling the grain drying process using neural networks.

\section{METHODS}

\section{Materials}

Corn grains (Zea mays) were used to identify the physiological response of agricultural grains product affected by the changing temperature during the drying process. Corn grains obtained directly from a farmer in Banjarnegara (149 meters above sea level), harvested after three months of cultivation. Corn grains from Banjarnegara were used because during the research conducted; it was enter harvesting period for corn grains in this area. Therefore, the sample for this research used fresh corn grains. Samples sent to the Laboratory of Agro-industrial Engineering, School of Applied Science, Universitas Gadjah Mada and preserve under ambient conditions during the experiment were conducted.

\section{The Water Loss Measurement System}

In this study, the physiological response of the grains was approached by monitoring the rate of water loss of grain during the drying process. By identifying the weight loss of the grains, the rate of water loss can be estimated. The grains weight loss continuously measured by an electronic balance OHAUS Scout Pro SKX2202 (OHAUS Corporation, New Jersey, USA) with the readability of $0.01 \mathrm{~g}$. Measurement conducted inside a drying chamber MEMMERT UN55 (Memmert GmbH + Co. KG, Germany) with the range of $40^{\circ} \mathrm{C}$ to $70^{\circ} \mathrm{C}$ of temperature treatment was chosen for the operations to identify the response of the rate of weight loss as affected by drying temperature. In order to prevent the excessive heat during the drying process that can affect the measurement accuracy due to the sensitivity of load sensor of the electronic balance, a $6 \mathrm{~mm}$ heat shield was applied to cover the electronic balance.

Sample of $1 \mathrm{~kg}$ of corn grains were used and composed as a $20 \mathrm{~mm}$ layer placed in a stainless tray $(215 \times 295 \times 60 \mathrm{~mm})$. The temperature inside the chamber and the weight loss of the grains during the experiment were continuously acquired through realtime data acquisition, with the sampling time was 30 seconds and monitored using a personal computer.

\section{Non-linear Autoregressive with Exogenous Input (NARX) for Dynamic Identification}

Neural networks were developed for identifying the dynamic response of the rate of water loss in the grains as affected by the drying temperature and for developing a black-box model simulation. By mimicking the biological human learning process, neural networks identifying the non-linear relationship between input and outputs of a system with their high learning abilities (Almási et al., 2016). Neural networks are a very useful approach based modeling method due to the traceback procedure ability during the training process (Ekhwan et al., 2009). The development of neural networks has been mature recently, one of the proven neural networks development which showed an excellent performance and has been widely applied in solving various types of complex nonlinear model problems is NARX (Chan et al., 2015; Mai et al., 2016; Mohd and Aziz, 2016; Darus et al., 2014; Yumeina et al., 2017). Lin et al. (1996) also reported that a NARX neural network has a better performance compared with recurrent neural networks trained by Backpropagation through time (BPTT).

Figure 1 shows the black-box system identification for modeling the single-input and single-output system. In this experiment, the drying temperature $T_{w}(k)$ is defined as the input variable, and the output variable is the rate of water loss of corn grains $W_{L}(k)$. The blackbox system identification is constructed from a NARX neural network model which consists of three different 


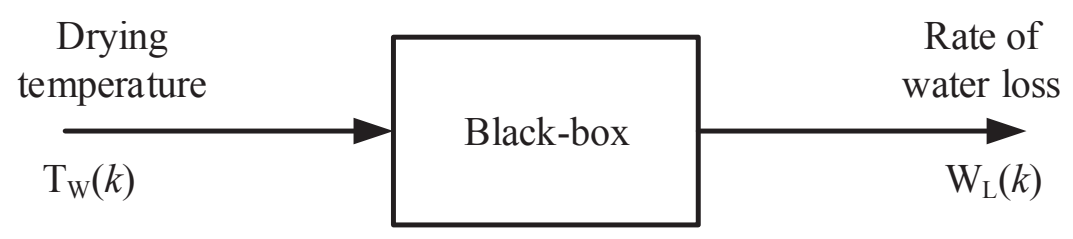

Figure 1. Single-input and single-output system

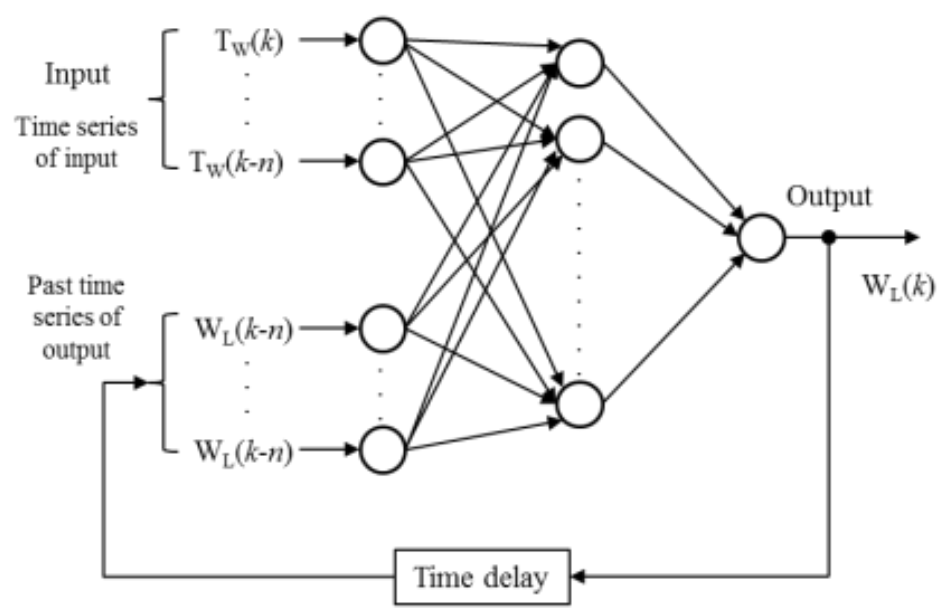

Figure 2. Structure of three layers of neural networks with time delay operator

layers: an input layer, a hidden layer and an output layer; and applying time delay operators to the input and output signal that produce time histories of the data for dynamic identification, as shown in Figure 2.

It is a non-linear function to estimate the output $\mathrm{W}_{\mathrm{L}}(k)$ at time sample $k$. For the learning or identification of neural networks, the $(n+1)^{\text {th }}$ historical input data, $\left\{\mathrm{T}_{\mathrm{w}}(k), \ldots, \mathrm{T}_{\mathrm{w}}(k-n)\right\}$ and the $\mathrm{n}^{\text {th }}$ historical output data, $\left\{\mathrm{W}_{\mathrm{L}}(k-1), \ldots, \mathrm{W}_{\mathrm{L}}(k-n)\right\}$, are used to the input layer, and current output $W_{L}(k)$ is applied to the output layer as training signals $(k=0,1, \ldots, N-n, N$ : number of data points) (Isermann et al., 1998). While the learning (training) method is using the Bayesian regularization (Beale et al., 2016). A dynamic model is obtained from the changes of weights and biases of the neural networks with the result that squared error between the network output and the training signal is minimized.

$W_{L}(k)=f\left(T_{W}(k), T_{W}(k-1), \ldots, T_{W}(k-n), W_{L}(k-1), \ldots, W_{L}(k-n)\right)$

For the prediction, the current output, $\mathrm{W}_{\mathrm{L}}(k)$, is estimated from two $(n+1)^{\text {th }}$ historical input data, $\left\{\mathrm{T}_{\mathrm{w}}(k), \ldots, \mathrm{T}_{\mathrm{w}}(k-n)\right\}$ and the $n^{\text {th }}$ historical output data, $\left\{\mathrm{W}_{\mathrm{L}}(k-1), \ldots, \mathrm{W}_{\mathrm{L}}(k-n)\right\}$, similar to an auto-regressive moving average (ARMA) model procedure (Isermann et al., 1998). The development made in this study was implemented using MATLAB 2015a computeraided design software. MATLAB ${ }^{\circledR}$ Neural Networks Toolbox $^{T M}$ version R2015a (MathWorks ${ }^{\circledR}$ Inc., Natick, Massachusetts, USA) (Beale et al., 2016) was utilized to develop the model with the code was set up to include all the above procedures.

\section{Model Validation}

The data sampled were divided into two data sets, which are a data set for training the neural networks, and the rest was a test data set for evaluating the accuracy of the model. The testing data set have to be independent of training data sets. The equal proportion is desirable for the number of data sets of training and testing. This type of model is called 'cross-validation' (Morimoto and Hashimoto, 2009).

\section{Model Selection}

The model performance was evaluated by mean squared error (MSE) and coefficient of determination $\left(R^{2}\right)$ as shown in Equation 2 and Equation 3, respectively.

$$
\begin{aligned}
& M S E=\frac{1}{n} \sum_{i=1}^{n}\left(y_{i}-\hat{y}_{i}\right)^{2}, \\
& R^{2}=\frac{\left(n \sum_{i=1}^{n} \hat{y}_{i} y_{i}-\sum_{i=1}^{n} \hat{y}_{i} \sum_{i=1}^{n} y_{i}\right)^{2}}{\left(n \sum_{i=1}^{n} \hat{y}_{i}{ }^{2}-\left(\sum_{i=1}^{n} \hat{y}_{i}\right)^{2}\right)\left(n \sum_{i=1}^{n} y_{i}{ }^{2}-\left(\sum_{i=1}^{n} y_{i}\right)^{2}\right)}
\end{aligned}
$$


where $n$ is the number of data points, is the actual value or network output, and is predicted value or the network target. The networks configuration selected by evaluating the value of MSE (the closer to zero, the better model performance prediction) and the value of (the closer to one, is the better model fits) (Kuhn and Johnson, 2013).

\section{RESULTS AND DISCUSSIONS}

\section{Identification of the Response of the Rate of Water Loss of Grains}

Fundamental water loss response of the grains to the temperature change during the drying process was successfully observed using the developed measurement system. Figure 3 shows a typical response of water loss of grains as affected by drying temperature. The drying temperature was initiated at $40^{\circ} \mathrm{C}$; several set points were applied to the system before it set to the $50^{\circ} \mathrm{C}$. When the grains were exposed to a higher temperature at $70^{\circ} \mathrm{C}$, the response of water loss at first rose before it steady maintaining the position following the temperature set. However, the response of water loss began to rise even when the temperature was maintained constant. Later, when the temperature was decreased to $50^{\circ} \mathrm{C}$, a slight delay or lags of the response of water loss happened before it began to fall until the minimum position and then rose again to the steady position. These uncertain and lags responses of water loss, suggest the complexities of dynamic responses of the water loss of grains as affected by the drying temperature is exist. Then, the observed dynamic responses of the grains during temperature stress were used for system identification.

\section{Prediction Performance Analysis}

The finding of the fittest model for the rate of water loss of grains during the drying process was generated from the simultaneous training procedure that running until the performance parameter reached (Beale et al., 2016). According to the Bayesian regularization algorithm, evidence procedures provide an objective Bayesian criterion for stopping training, and it will prevent the over train process. As shown in Figure 4.a, the performance of the model is evaluated during training, from the graph, it shows the value of MSE of the model decrease as the weight is improved.

The prediction performance of the selected NARX neural networks model can also be analyzed by evaluating time series response, the autocorrelation of error and input error cross-correlation, as shown in Figure 4.b, $c$ and $d$ respectively. Figure 4.b shows the comparison of trained output value and the target or observed value. The lesser error between those values is better. Then autocorrelation of error in the Figure 4.c suggest the significance of error and its correlation to the time series prediction, while input-error crosscorrelation at the figure 4.d reference to the correlation of error to the time series input values (Beale et al., 2016). From the Figures 4.c and 4.d have shown that mostly the correlations fall within confidence bound,
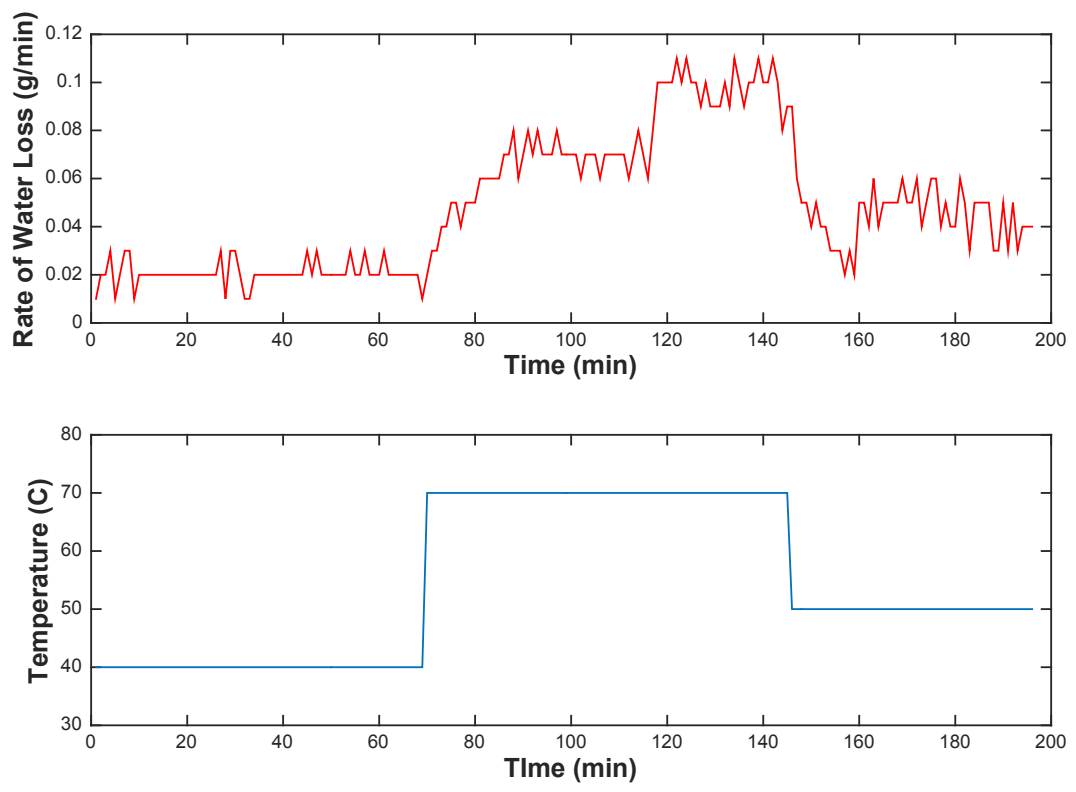

Figure 3. The relationship between drying temperature and the rate of water loss of the grains 


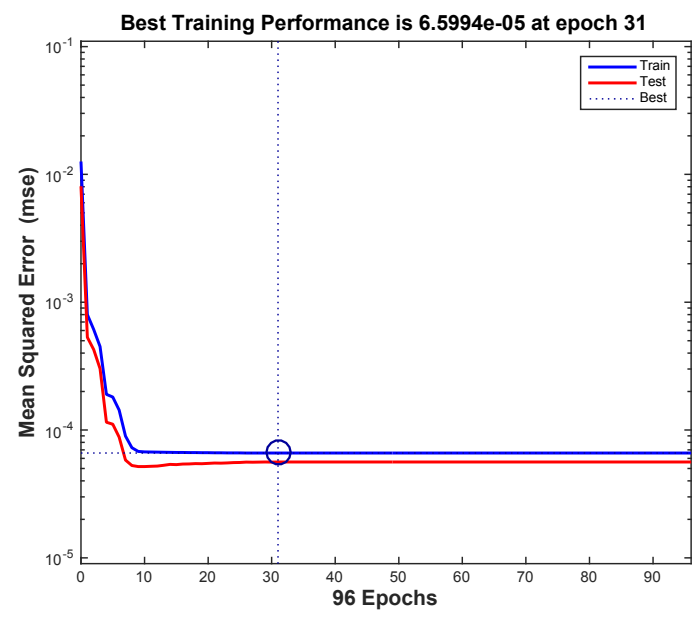

(a)

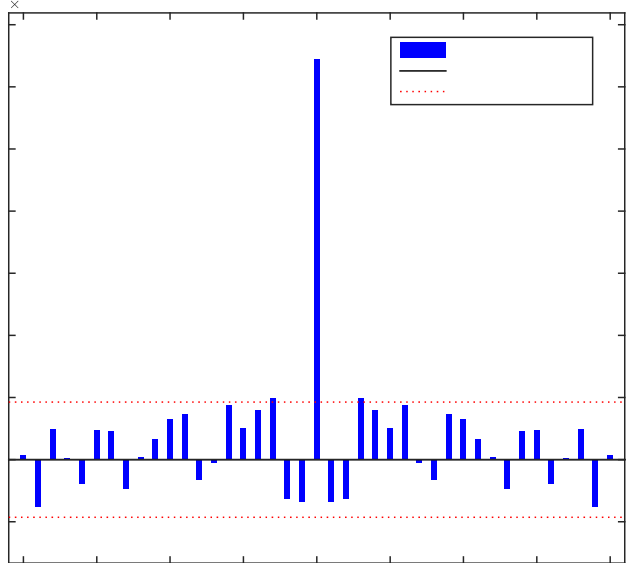

(c)

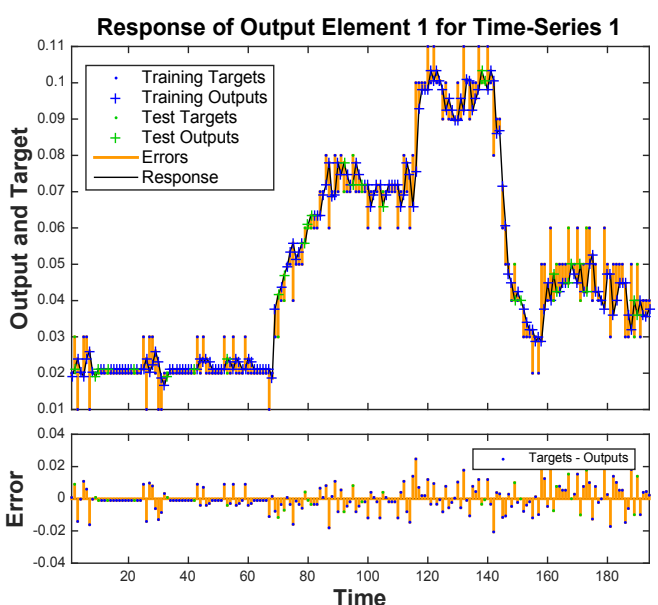

(b)

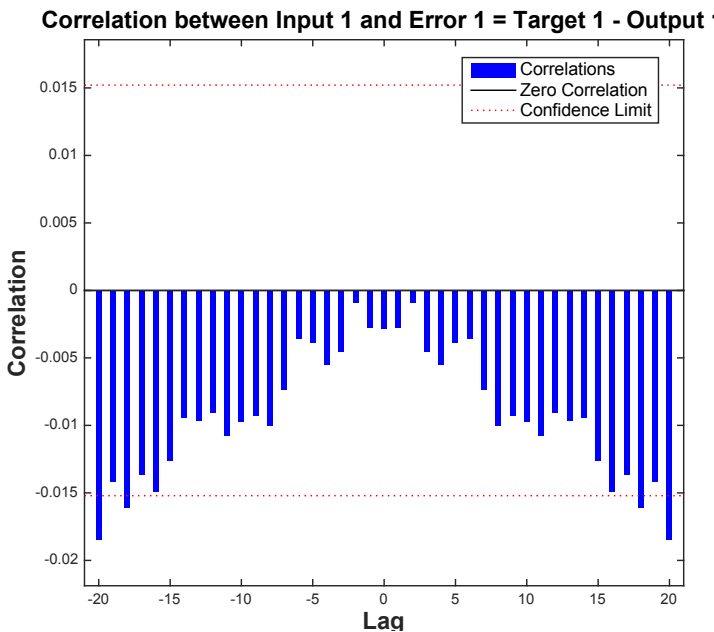

(d)

Figure 4. Prediction performance analysis of NARX model

this implies that selected NARX neural networks model was good enough and efficient in predicting the rate of water loss of corn grains with the drying temperature.

\section{Sensitivity Analysis}

Figure 5 shows the regression analysis of NARX neural networks model during training, test, and validation procedures. As the coefficient of determination $\left(R^{2}\right)$ value is close to one; it suggests that the NARX neural networks fit enough to represent the actual system, and also as a direct measure of sensitivity and the influence of drying temperature to the rate of water loss (Iooss and Lemaître, 2015). Therefore, the results imply that the developed NARX neural networks were well in predicting the rate of water loss of grains by drying temperature.
Through training and validation process, the combination of time step delay and number of neuron in the hidden layer determine the NARX neural network model performance. NARX neural networks with the combination of 1-10-1 neuron number in input, hidden, and output layer respectively, and with two times step delay $(\mathrm{d}=2)$ was selected because of its best performance. Figure 6 shows the comparison of the estimated and observed relationship between the rate of water loss of the grains and drying temperature. The estimated value was obtained from the simulation of the selected NARX neural networks model, with a testing data set is different from the training data sets was used. It can be seen that the estimated response was closely related to the observed response, with the coefficient of determination value $\left(R^{2}\right)$ is 0.89 , and the MSE is $1.89 \times 10^{-4}$. This result implies that NARX neural 


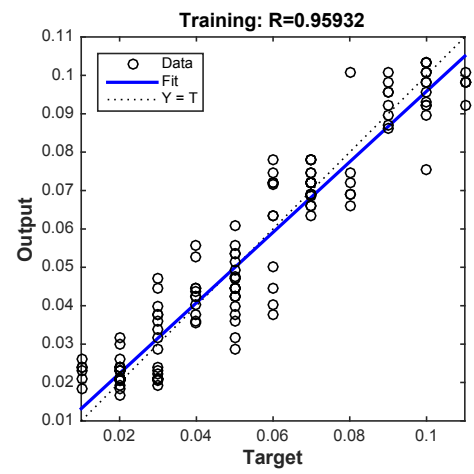

(a)

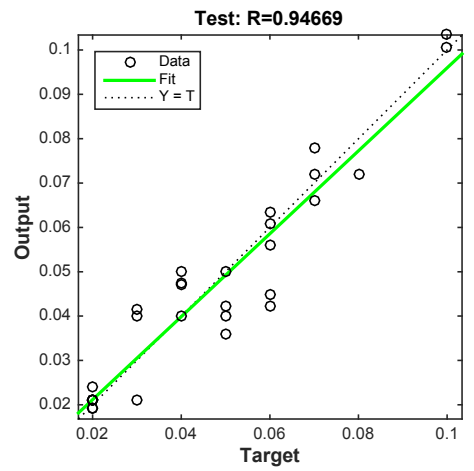

(b)

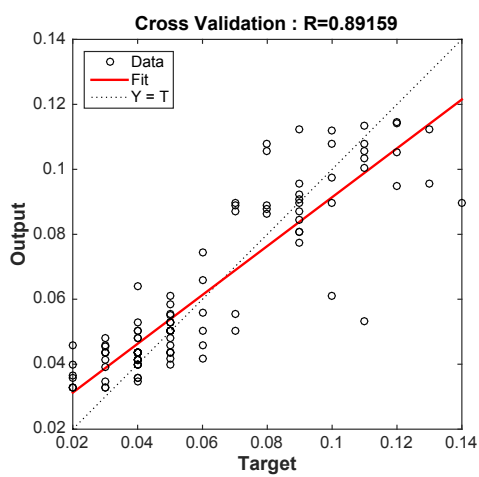

(c)

Figure 5. Regression analysis of NARX model development: (a) Training; (b) Test; and (c) Cross-Validation

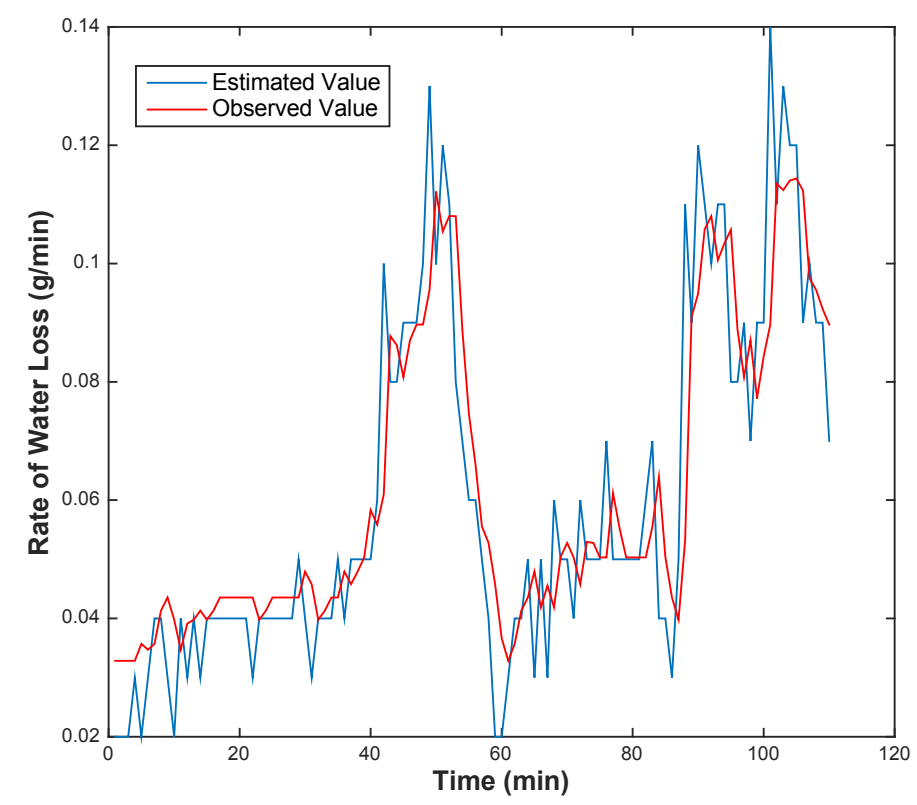

Figure 6. The simulated dynamic nonlinear relationship between the estimated and observed response of the grains during the drying process

networks work well to develop the dynamic model that deals with the nonlinear and complex character of the grains response and drying temperature.

\section{CONCLUSIONS}

In this study, a three-layered NARX neural networks (1-10-1) (input-hidden-output layer neuron number) with times step delay $(\mathrm{d}=2)$ has a high model approximation accuracy (MSE $=1.89 \times 10^{-4} ;=0.89$ ). It is also successful in identifying and developing a model such a complex system as the dynamic characteristics of the rate of water loss of grains to drying temperature. A NARX neural networks model obtained, provides an alternative and accuracy in predicting the rate of water loss of grains by drying temperature. This research also can be used as a basis to develop a further study on dynamic control optimization technique.

\section{ACKNOWLEDGMENTS}

The authors of this paper would like to acknowledge the program of Peningkatan Kapasitas Peneliti Dosen Muda Universitas Gadjah Mada 2017 for funding of this study, the cooperation of the research team and the Department of Bioresource Technology and Veterinary, the study program of Agroindustri, Vocational College Universitas Gadjah Mada for the support. 


\section{CONFLICT OF INTEREST}

There was no conflict interest of the article publication.

\section{REFERENCES}

Aghbashlo, M., Hosseinpour, S., \& Mujumdar, A. S. (2015). Application of Artificial Neural Networks (ANNs) in Drying Technology: A Comprehensive Review. Drying Technology. https://doi.org/10.1080/07373937.2015.10 36288

Almási, A.-D., Woźniak, S., Cristea, V., Leblebici, Y., \& Engbersen, T. (2016). Review of advances in neural networks: Neural design technology stack. Neurocomputing, 174, 31-41. https://doi.org/10.1016/J.NEUCOM.2015.02.092

Baloch, M. S., Morimoto, T., \& Hatou, K. (2006). Stress Application Loss of Fruits Technique during for Storage Reducing Water. Environmental Control in Biology, 44(1), 31-40.

Beale, M. H., Hagan, M. T., \& Demuth, H. B. (2016). Neural Network Toolbox (TM) User's Guide. MathWorks (R2018a ed.). The MathWorks, Inc. https://doi. org/10.1002/0471221546

Chan, R. W. K., Yuen, J. K. K., Lee, E. W. M., \& Arashpour, M. (2015). Application of Nonlinear-AutoregressiveExogenous model to predict the hysteretic behaviour of passive control systems. Engineering Structures, 85, 1-10. https://doi.org/10.1016/j.engstruct.2014.12.007

Dai, A., Zhou, X., \& Liu, X. (2017). A GODFIP Control Algorithm for an IRC Grain Dryer. Mathematical Problems in Engineering, 2017, 1-14. https://doi. org/10.1155/2017/1406292

Ekhwan Toriman, M., Ekhwan Toriman, M., Juahir, H., Mokhtar, M., Barzani Gazim, M., Mastura Syed Abdullah, S., \& Jaafar, O. (2009). Predicting for Discharge Characteristics in Langat River, Malaysia Using Neural Network Application Model. Research Journal of Earth Sciences, 1(1), 15-21.

Fasol, K. H., \& Jörgl, H. P. (1980). Principles of model building and identification. Automatica, 16(5), 505-518. https:// doi.org/10.1016/0005-1098(80)90074-6

Iooss, B., \& Lemaître, P. (2015). A review on global sensitivity analysis methods. Operations Research/ Computer Science Interfaces Series. https://doi.org/10.1007/9781-4899-7547-8_5

Isermann, R., Ernst, S., \& Nelles, O. (1998). Identification with dynamic neural networks - Architectures, comparisons, applications. (Sysid'97): System Identification, Vols 1-3, 947-972.
Kuhn, M., \& Johnson, K. (2013). Measuring Performance in Regression Models. In Applied Predictive Modeling. https://doi.org/10.1007/978-1-4614-6849-3_5

Lin, T., Horne, B. G., Tiňo, P., \& Giles, C. L. (1996). Learning long-term dependencies in NARX recurrent neural networks. IEEE Transactions on Neural Networks, 7(6), 1329-1338. https://doi.org/10.1109/72.548162

Ljung, L. (2010). Perspectives on system identification. Annual Reviews in Control, 34(1), 1-12. https://doi. org/10.1016/J.ARCONTROL.2009.12.001

Mai, C. V., Spiridonakos, M. D., Chatzi, E. N., \& Sudret, B. (2016). Surrogate modeling for stochastic dynamical systems by combining nonlinear autoregressive with exogenous input models and polynomial chaos expansions. International Journal for Uncertainty Quantification, 6(4).

Mohd, N., \& Aziz, N. (2016). Performance and robustness evaluation of Nonlinear Autoregressive with Exogenous input Model Predictive Control in controlling industrial fermentation process. Journal of Cleaner Production, 136, 42-50. https://doi.org/10.1016/j.jclepro.2016.06.191

Morimoto, T, Islam, P., Suyantohadi, A., \& Ouchi, Y. (2010). Dynamic optimization of watering for maximizing the sugar content and size of Satsuma mandarin using intelligent approaches. IFAC Proceedings Volumes (Vol. 43). https://doi.org/10.3182/20101206-3JP-3009.00043

Morimoto, T, \& Hashimoto, Y. (2009). Speaking plant/ fruit approach for greenhouses and plant factories. Environmental Control in Biology, 47(2), 55-72.

Ng, B. C., Darus, I. Z. M., Jamaluddin, H., \& Kamar, H. M. (2014). Dynamic modelling of an automotive variable speed air conditioning system using nonlinear autoregressive exogenous neural networks. Applied Thermal Engineering, 73(1), 1253-1267. https://doi. org/10.1016/j.applthermaleng.2014.08.043

Rumelhart, D. E., Hinton, G. E., \& Williams, R. J. (2013). Learning Internal Representations by Error Propagation. In Readings in Cognitive Science: A Perspective from Psychology and Artificial Intelligence (pp. 399-421). https://doi.org/10.1016/B978-1-4832-1446-7.50035-2

Yumeina, D., Aji, G. K., \& Morimoto, T. (2017). Dynamic optimization of water temperature for maximizing leaf water content of tomato in hydroponics using an intelligent control technique. Acta Horticulturae (Vol. 1154). https://doi.org/10.17660/ActaHortic.2017.1154.8 